A N N A L E S Annales de Bretagne et des Pays de l'Ouest

\title{
Le Dauphin de 1815 : étude d'une rumeur
}

\section{Yann Guerrin}

\section{OpenEdition}

\section{Journals}

Édition électronique

URL : http://journals.openedition.org/abpo/1419

DOI : $10.4000 / a b p o .1419$

ISBN : 978-2-7535-1490-4

ISSN : 2108-6443

Éditeur

Presses universitaires de Rennes

Édition imprimée

Date de publication : 20 juillet 2003

Pagination : 111-128

ISBN : 978-2-86847-874-0

ISSN : 0399-0826

\section{Référence électronique}

Yann Guerrin, "Le Dauphin de 1815 : étude d'une rumeur », Annales de Bretagne et des Pays de l'Ouest [En ligne], 110-2 | 2003, mis en ligne le 20 juillet 2005, consulté le 19 avril 2019. URL : http:// journals.openedition.org/abpo/1419; DOI : 10.4000/abpo.1419 


\title{
Le Dauphin de 1815 : étude d'une rumeur
}

\author{
Yann GUERRIN \\ Docteur en histoire, lycée Mariette, Boulogne-sur-Mer
}

\section{L'arrestation d'un vagabond}

En décembre 1815, un singulier personnage est interpellé pour vagabondage à Saint-Malo. Intriguant la police, il est arrêté et interrogé. Il déclare provenir d'Amérique et ajoute que le consul des États-Unis à Nantes lui aurait remis la somme de 720 francs pour couvrir les frais de son retour vers le Nouveau Monde. Somme qui lui aurait été dérobée par un capitaine de barque allant à Jersey. L'inconnu devient vraiment suspect lorsqu'il affirme avoir perdu ses papiers. Il est immédiatement placé en détention administrative le temps d'une vérification de routine. Son signalement permet d'imaginer la physionomie du personnage : 33 ans, 1,67 m, châtain aux yeux bruns clairs, nez un peu aquilin tourné à droite, une fossette en bas de la joue gauche, le visage ovale et plein ${ }^{1}$. Le commissaire de police reçoit très rapidement confirmation de ses soupçons : le représentant des ÉtatsUnis en question n'a jamais entendu parler de cette étrange histoire! Cependant, le suspect ne s'affole nullement, prend " un tout autre ton " et se présente alors comme "Charles de Navarre, Dauphin ". Il affirme pour la première fois, et pour longtemps, qu'il est le fils de Louis XVI, pourtant officiellement mort en juin 1795! Pour les autorités, il ne fait aucun doute que cet homme est fou et ne mérite aucune attention particulière. Le comte de Viomeuil gouverneur de la $13^{\mathrm{e}}$ division militaire, expédie alors la nouvelle de l'arrestation et ses déclarations au préfet "sans juger d'ailleurs qu'elles ne méritent aucune importance ${ }^{2}$ ".

Les circonstances et les aspirations populaires auront tôt fait de le démentir car l'affaire du Dauphin de Saint-Malo prend des proportions extraordinaires en un temps inouï. Elle nous place devant un cas exemplaire de rumeur politique qui est le fruit d'une époque, d'une population et d'un individu hors du commun. L'affaire est connue sous le nom de " affaire Mathurin Bruneau " et figure dans de nombreux ouvrages répertoriant les

1. Le sous-préfet à Saint-Malo, le 19 décembre 1815, Arch. dép. d'Ille-et-Vilaine 4 M 88 2. Ibid. 
non moins nombreux prétendants au titre de Louis XVII ${ }^{3}$. Cependant, jamais la question ne fut étudiée sous l'aspect de la rumeur. Elle saisit une ville puis une région et enfin tout un pays en quelques semaines pour s'étendre sur plusieurs années. Comment de simples bruits peuvent-ils acquérir autant de force, être avidement transmis, passionnément commentés par toute une société en même temps que craints au plus haut niveau de l'État?

\section{La naissance d'une affaire d'État}

De nos jours, ce qui ne manque pas d'attirer l'attention, c'est le contraste entre le mépris des autorités et la fulgurante diffusion des propos du prisonnier. Comme pour toute rumeur de grande ampleur, il s'agit avant tout de la rencontre d'une information avec un public. L'adéquation est ici exemplaire. La police et les autorités civiles de Saint-Malo qualifient tout d'abord d'aventurier celui qui prétend être Charles de Navarre, fils de Louis XVI et neveu de Louis XVIII. Le sous-préfet rassure son supérieur hiérarchique à la préfecture de Rennes en écrivant qu'il considère cet homme " comme un fripon et un fou [dont les] manières sont communes, [le] langage pitoyable, [les] raisonnements nuls ${ }^{4}$ ». Il raille les maladresses de son expression qui l'éloignent de la majesté royale à laquelle il ose prétendre. "Il parle assez mal : il dit par exemple, ils tombirent pour ils tombèrent faute de langage assez commune chez le peuple dans ces contrées et ses environs. " Il ne fait alors aucun doute que l'aventurier ne tardera pas à être démasqué. Deux hypothèses se partagent les faveurs de l'administration départementale : la folie ou l'imposture. Tous les rapports officiels émanant des autorités locales insistent sur le peu de gravité d'une affaire plus pittoresque qu'inquiétante.

Or, tandis que les autorités rédigent des rapports rassurants, la nouvelle court les rues et se répand à tous les niveaux de la société. La spontanéité et la rapidité de la diffusion suffisent à démontrer la puissance de cette rumeur. Comme souvent, nous ne pouvons l'approcher qu'au travers des rapports et commentaires des officiels. Parmi la population, personne ne songe à en consigner l'évolution. L'émotion que suscite la nouvelle balaie toute réflexion et toute prise de recul. La rumeur est en effet une nouvelle invérifiable à " consommer " aussi vite que possible. Celui qui la reçoit avant les autres peut jouer d'importance en captivant l'attention de son entourage. Pour un temps! L'information "rumeurogène " prodigue un pouvoir de séduction intense mais fugace. C'est une denrée précieuse mais éphémère, à diffuser aussi vite que possible pour ne pas perdre l'avantage de la primeur. La véracité de ce genre d'information compte moins que sa

3. Les Archives départementales d'Ille-et-Vilaine ont répertorié ce dossier sous la cote 4 M 88. Aux Archives nationales F/7/6979 et BB 18979 dossier Mathurin Bruneau, pièce $\mathrm{n}^{\circ} 65$.

4. Arch. dép. d'Ille-et-Vilaine, 4 M 88. 
capacité de séduction. Il faut songer qu'en cette fin d'année 1815, la nouvelle est de taille : le fils de Louis XVI dans les prisons de Saint-Malo! Ce serait la fin d'un mystère! Ce serait une affaire d'État dans les murs d'une ville provinciale, si éloignée de Paris! Ce serait l'occasion d'apercevoir un enfant présumé mort en sa dixième année! Il en faut moins pour affoler toute une ville!

Louis-Charles, fils de Louis XVI et de Marie-Antoinette, est né à Versailles le 27 mars 1785. La famille royale est emprisonnée à la prison du Temple à partir du 13 août 1792. Le jeune héritier est alors âgé de 7 ans. Pendant ses trois années de détention, il assiste impuissant à la condamnation et à l'exécution de ses parents. Son éducation est confiée au cordonnier sansculotte : Antoine Simon. De janvier à juillet 1794, l'enfant est enfermé dans une cellule, seul, sans aucun contact avec l'extérieur. Gravement affecté physiquement et mentalement par ce traitement inhumain, l'héritier de la couronne de France meurt officiellement le 8 juin 1795. Le destin de cet enfant est proprement extraordinaire car l'infortuné est un prisonnier reconnu par toute l'Europe comme héritier de la couronne de France dès le 28 janvier 1793. Les armées royales de l'Ouest émettent des documents officiels au nom de Louis XVII. Son effigie figure sur d'innombrables supports circulant clandestinement. Rien n'arrête la tradition et, à sa mort, son oncle le comte de Provence, prend le titre de Louis XVIII succédant ainsi à celui qui n'a jamais régné que depuis sa cellule. C'est cette version officielle que le prétendant de Saint-Malo vient bousculer. Ses prétentions accréditent alors la piste d'une évasion du jeune roi de sa geôle et d'une substitution de corps ${ }^{5}$. En 1815, il aurait trente ans. Son retour constituerait la fin d'une énigme, un rebondissement dynastique, un happy end pour l'un des épisodes les plus sombres de la Révolution. Ce serait par la même occasion une affaire d'État aux graves conséquences politiques en même temps qu'un élément de réconciliation nationale entre Bleus et Blancs. En tout état de cause, la nouvelle fait l'effet d'une bombe!

La diffusion par la puissance du bouche à oreilles est l'affaire de quelques heures à Saint-Malo et dans ses environs. La police commet en effet une série de bévues qui relèvent du manque de prudence à une époque et dans une société où le moindre bruit peut enfler pour échapper à tout contrôle. Ces négligences en disent long sur les erreurs d'appréciation des autorités. Sans que personne ne puisse expliquer qui en a donné la consigne, une foule se réunit le 19 décembre entre 16 et 18 heures. Elle cherche à en savoir plus sur celui que l'on espère déjà être Louis-Charles, fils de Louis XVI et de Marie-Antoinette. La police évalue ce rassemblement à " quatre cents personnes au moins autour de la prison pour le voir ". Il faut la mobilisation d'une vingtaine de soldats pour disperser l'attroupement. Cette intervention conforte ceux qui pensent que les autorités ont

5. Pour mieux comprendre les arguments des survivantistes et des spécialistes voir : GREY, Marina, Enquête sur la mort de Louis XVII, Paris, Librairie académique Perrin, 1989; PInCEMAILle, Christophe, "L'affaire Louis XVII ", L'Histoire, 1989, n 122, p. 48-53. 
quelqu'un d'important ou de gênant à cacher. En se retirant, la foule profère déjà quelques cris : "Vive le Roi et Charles de Navarre ${ }^{6}$. " Le lieutenant de police précise dans son rapport que « le bruit s'était répandu qu'on allait traduire le détenu devant le juge d'instruction ou le transférer ${ }^{7}$ ". La foule des curieux est clairement venue pour voir cet homme dont l'histoire a manifestement filtré hors des murs de la prison. L'origine des fuites ne peut être recherchée que parmi les policiers, militaires ou geôliers, c'est-à-dire dans le premier cercle d'initiés. Ont-ils seulement reçu des consignes de discrétion? Rien ne l'indique car aucune enquête interne ne mentionne la moindre précaution destinée à garder le secret. Pourtant, sans que nul n'y prête attention une rumeur sort de l'œuf! Lors de ce rassemblement populaire, un incident décuple les effets de la rumeur naissante : un prisonnier au courant de ce qui se joue devant la prison, accroche à la fenêtre de sa cellule (donnant sur la rue), un tissu blanc en guise de drapeau. Il va même jusqu'à jeter des pièces de menue monnaie sur la foule qui interprète aussitôt ce geste comme un signe (probatoire) de la générosité des Bourbons. Cet épisode accrédite un peu plus la rumeur sur la qualité du suspect (même s'il n'y est pour rien). Dès lors, l'un des mécanismes de la rumeur est en action : tous les indices ou signes susceptibles de la conforter sont récupérés. Ils lui procurent toujours plus de force.

Deux jours après son arrestation, le prétendu Charles de Navarre est promené dans les rues de la ville au mépris des règles les plus élémentaires de la sécurité et en vertu d'une méconnaissance remarquable de la psychologie des foules. La police lui fait traverser la ville pour un interrogatoire ainsi que pour le faire identifier par des personnes ayant déclaré le connaître. Immédiatement, ses gardiens remarquent que la population est manifestement impressionnée par ses manières et son allure. Cette promenade anodine sert considérablement sa cause : "Elle a accrédité dans le vulgaire les dires de cet aventurier. " Le public est loin d'être déçu par le personnage dont toute la ville parle depuis deux jours. Son allure, sa physionomie correspondent à l'imaginaire populaire. Malgré la modestie de ses vêtements, il correspond à l'image que l'on se fait d'un prince royal. Beaucoup lui reconnaissent une ressemblance avec les plus éminents représentants de la famille Bourbon. Notons que peu nombreux sont ceux qui ont eu l'occasion d'en approcher un. Qu'importe! Le temps d'une promenade, le cercle de ses partisans se trouve considérablement élargi. Désormais, il ne fait pas de doute que la rumeur trouve écho dans un large public qui peut personnaliser le prétendant et bavarder à l'infini à son sujet : ce jour-là une rumeur est née!

Au matin du 22 décembre, le préfet croit pouvoir écrire au ministre de la police que la rumeur est encore circonscrite à Saint-Malo et Saint-Servan. Mais à partir du 23, tout le département est touché par l'intermédiaire des poissonniers malouins qui colportent cette nouvelle extraordinaire. Les

6. Arch. dép. d'Ille-et-Vilaine, 4 M 88

7. Ibid. 
révélations suscitent d'incontrôlables commentaires. Dans un rapport daté du 24 décembre, le sous-préfet de Fougères s'en alarme écrivant au préfet que « des poissonniers ont répandu à Fougères la nouvelle hier matin, jour de marché, de l'apparition à Saint-Malo d'un homme se disant le fils de Louis XVI ». Un voyageur le confirme au préfet :

" Un peuple énorme le suivait quand on le menait dans les rues de SaintMalo. Les magistrats ne croyaient pas, ajouta-t-il, que ce fut le fils du roi, mais les gens du peuple étaient portés à le croire; en général cette classe est amie du merveilleux. À Fougères, cette nouvelle a été la plus courue du marché ${ }^{8}$."

Les autorités comprennent alors tout le danger que ces bruits comportent. Mais il est trop tard pour la contrôler. Le préfet réalise qu'il est particulièrement difficile de lutter. La rumeur a acquis une force suffisante pour courir les chemins et les routes. Son contenu s'est lentement précisé. Il était très faible au début de l'affaire mais il s'enrichit de tous les indices : la rumeur fait feu de tout bois! Un suspect, des déclarations sans preuve, un mouchoir blanc, un prisonnier promené dans les rues ont suffit à la population. Elle élabore rapidement un contenu qui renforce les prétentions du détenu. Il ne faut que quelques jours pour que la déclaration échappe à sa source. La rumeur s'est stabilisée autour d'une version assez simple pour être diffusée, en même temps qu'assez complexe pour être convaincante auprès du plus grand nombre. L'accentuation constitue l'évolution suivante habituelle : pour conserver son pouvoir mobilisateur auprès du premier cercle des initiés, des détails sont sans cesse ajoutés. Ainsi, les sujets de conversation tourneront-ils sans relâche autour du même thème mais avec d'innombrables variations. Parmi les ajouts d'accentuation, peu nous sont parvenus mais le sous-préfet de Vitré annonce que, dans son arrondissement, la rumeur ajoute que des princes de la famille royale circulent incognito pour soustraire le prisonnier de sa prison. Sans tarder, le préfet d'Ille-et-Vilaine s'inquiète. La puissance de la rumeur est si importante qu'elle dépasse bientôt le département pour saisir la Bretagne puis tout le pays.

\section{Les mécanismes de la rumeur}

Avant d'étudier plus profondément les éléments moteurs de cette singulière affaire, sans doute convient-il de réfléchir à la notion de rumeur. Force est de constater que ce mot couvre des réalités très différentes. Généralement, les rumeurs sont considérées comme des bruits au contenu plutôt mal défini et qui circulent sans logique parmi la population. Elles seraient quantité négligeable, aussi vite nées que disparues. Généralement, elles sont reçues comme mystérieuses et négatives, en quelque sorte comme un " marché noir de l'information ${ }^{9}$ ". Elles seraient proches des ragots et des potins.

8. Ibid. Le sous-préfet de Fougères au préfet, le 24 décembre 1815.

9. KAPFERER, Jean-Noël, Rumeur, le plus vieux média du monde, Paris, Seuil, 1987, 317 p. 
Elles sont bien plus que cela. Des études précises ont démontré qu'elles répondent à des règles et des logiques propres. Les premières études systématiques concernant ce mode d'expression remontent à la Première Guerre mondiale ${ }^{10}$. Ce sont surtout les Américains qui leur consacrent des études de grande ampleur pendant la Seconde Guerre mondiale. En 1944, R. Knapp avance une explication large : la rumeur est " une déclaration destinée à être crue, se rapportant à l'actualité et répandue sans vérification officielle ${ }^{11}$ ". La définition de Allport et Postman reste assez proche : "Une affirmation générale présentée comme vraie, sans qu'il existe de données concrètes, permettent de vérifier son exactitude ${ }^{12}$. " Trente ans plus tard, M.-L. Rouquette complète et complexifie. Pour lui, trois conditions déterminent l'existence d'une rumeur : la rumeur s'oppose à l'écrit en ce que sa diffusion est principalement orale, passant d'un individu à un autre individu; elle apporte des renseignements sur une personne ou sur un événement, parfois les deux; elle exprime et satisfait les besoins émotionnels des individus. Bref, elle répond à une attente ${ }^{13}$. J.-N. Kapferer réfute les approches idéologiques, moralisantes et psychiatrisantes ${ }^{14}$. Il reçoit la rumeur comme une action collective en vue de donner un sens à un fait inexpliqué. Cette approche plus large que les précédentes s'accorde avec le cas étudié ici. La rumeur peut s'appuyer sur des informations aussi bien vraies que fausses. Il convient qu'elle soit plausible. Partout où le public s'interroge et cherche à comprendre sans recevoir de réponses émanant des autorités officielles, la rumeur offre une alternative. Le contenu d'une rumeur correspond presque toujours à un point de départ : événement mal interprété ou mal compris, mauvaise foi, propos déformés ou malveillants... Dans le cas présent, elle naît de la rencontre d'une énigme historique, des fantasmes d'une population traumatisée par la mort d'un héritier royal et des déclarations d'un suspect : mélange propice à toutes les conjectures. La rumeur renvoie aux interrogations, aux angoisses, aux frustrations d'une société à un moment historique identifiable. Elle démontre des capacités d'adaptation importantes : " La logique de la rumeur n'est pas celle de la raison. Pour exister, il lui suffit de circuler, d'être sans cesse en mouvement; elle se nourrit d'elle-même ${ }^{15}$. " Aucune définition courte ne peut rendre compte de son extraordinaire variété. Elle peut prendre sa source dans une information fausse ou avérée. Elle constitue un mode de communication finalement assez

10. DaUZAT, A., Les Faux bruits et les légendes de guerre; GraUX, L., Les Fausses nouvelles de la Grande guerre; SELLERS, E., On Fighting against lying rumours.

11. KnAPP, Robert, "A psychology of rumor ", Public opinion quaterly, 8, 1944.

12. Allport, Gordon W., Postman, Leo, The Psychology of rumor, New York, 1947.

13. Michel-louis RouQueTte, Les rumeurs, Paris, Presses universitaires de France, 1975, $126 \mathrm{p}$.

14. Edgar MoRin dans La Rumeur d'Orléans, donne de la rumeur l'image d'une maladie collective contre laquelle il convient de réagir. Si celle qu'il étudie prend des allures antisémites, il ne faut pas oublier qu'il ne s'agit que d'une étude de cas. Toutes les rumeurs ne doivent pas être considérées comme une déviance.

15. FLEM, Lydia, "Bouche bavarde et oreille curieuse ", Le Genre Humain : La rumeur, n 5, éd. Complexe, 1982, p. 13. 
commun pour une population, tout particulièrement pendant les périodes de sous-information, d'angoisse ou de crise. N'oublions pas que sur une population d'à peine trente millions d'habitants au début de la Restauration, $80 \%$ sont des illettrés ${ }^{16}$. Elle circule sans contrainte, sans autre logique que les rencontres dans les rues ou les lieux publics. À la seule condition d'être crédible, elle peut être transmise par n'importe qui. La Grande Peur de l'été 1789 étudiée par Georges Lefèvre montre la force de nouvelles alarmistes au sein d'une population réceptive ${ }^{17}$. La rumeur a des vertus d'adaptation et de souplesse ${ }^{18}$; la moindre nouvelle peut être soumise aux exagérations et aux interprétations. La plupart des rumeurs qui nous sont connues ont une ampleur régionale ou nationale. Mais il ne faut pourtant pas oublier les innombrables rumeurs qui s'attachent à une personnalité locale et n'intéressent qu'une ou plusieurs communautés villageoises. La majorité des rumeurs ne concerne qu'un groupe social réduit mais certaines ont une portée beaucoup plus vaste. Il faut surtout retenir qu'elle échappe aux canaux de l'information officielle, qu'elle naît et se diffuse spontanément de bouche à oreille et à grande vitesse. Elle permet d'approcher les attentes, les frustrations, les craintes de ceux dont généralement l'Histoire ne retient pas les paroles. Disons plus simplement qu'il s'agit du mode de communication populaire par excellence car ne nécessitant aucun moyen technique et répondant instantanément aux interrogations collectives.

De nos jours, le succès de cette rumeur ne peut être compris qu'en recourant à plusieurs niveaux d'explications. Elle apparaît d'abord comme le fruit d'une époque. À partir de 1815, le contexte politique est placé sous le signe de la confusion et de la fragilité. La restauration des Bourbons en la personne de Louis XVIII, frère de Louis XVI, a été fragilisée par l'épisode des Cent Jours. La question de la légitimité du régime se pose cruellement. Ce monarque sait que s'il dispose de partisans, il doit pourtant compter avec un pays profondément modifié par la Révolution et l'Empire. Écrasé entre les aspirations libérales et ultraroyalistes, il doit trouver un équilibre. Il succède à son frère Louis XVI, guillotiné, et à son neveu mort au Temple. À ceci, s'ajoute la double défaite militaire de 1814 et 1815 . Entre héritage révolutionnaire et tradition d'Ancien Régime, le nouveau roi a conscience de la fragilité de sa position. Sa fuite pendant les cent-Jours n'a pas arrangé les choses. Nombre de Français raillent le "roi de Gand " revenu en France dans les fourgons des armées étrangères. La crise frumentaire de 1817 soumet les couches populaires à des tensions tout à fait propices à la diffusion de rumeurs diverses. Tout ceci demande des explications que la rationalité ne parvient pas toujours à fournir.

16. Cité par Michel-Louis RouQuetTe, La Rumeur et le meurtre : l'affaire Fualdès, Paris, Presses universitaires de France, 1992, 110 p.

17. LefeVre, Georges, La Grande Peur de 1789, Paris, A. Colin, 1988, 271 p.

18. Pour une définition encore différente, consulter, TruxiLLo Jean-Paul, CoRso, Philippe, Dictionnaire de la communication, Paris, A. Colin, 1991, 591 p. et Ploux, François, De bouche à oreille. Naissance et propagation des rumeurs dans la France du XIXe siècle, Paris, Aubier, 2003, 289 p. 
D'autre part, la France de 1815 ne ressemble plus tout à fait à celle d'avant 1789. Les rebondissements des années 1814 et 1845 ont confronté le pays à l'instabilité politique : double retour des Bourbons, Cent jours, défaites puis invasions. Dans son ouvrage, Les Vingt jours, Jean Tulard étudie les hésitations de ceux qui doivent se prononcer en faveur de deux légitimités qui s'opposent en mars $1815^{19}$. L'opinion publique sort profondément ébranlée de cette période tumultueuse : fidélités, serments, certitudes ont été soumis à rude épreuve. Le Dictionnaire des girouettes ${ }^{20}$ est d'ailleurs le plus grand succès de librairie de cette époque de ralliements hâtifs et de trahisons. C'est l'aveu d'une perte de repères, de valeurs et de confiance dans des élites compromises par des nombreux revirements.

En marge de cette recomposition du personnel dirigeant et des élites, la perception des rythmes politiques change. Sous l'Ancien Régime, la question de la légitimité politique ne se pose pas puisque les règles de succession sont immuables et parfaitement réglées par la tradition. Ceci a garanti une longue stabilité au pays que les bouleversements politiques inaugurés en 1789 peuvent faire regretter à certains en 1815. La Révolution inaugure en effet le temps des changements brutaux. Une quinzaine d'années plus tard, les mentalités collectives ont nettement évolué. Le peuple a été confronté à des questions inconnues jusque-là : avec Bonaparte, c'est l'image de l'homme neuf et providentiel qui s'oppose à la continuité des Bourbons. Le goût du coup de théâtre politique s'impose durablement dans l'opinion publique française du XIX ${ }^{\mathrm{e}}$ siècle. François Furet écrit qu'il existe désormais dans la population le sentiment "d'une fatalité de la répétition des scènes et des régimes ${ }^{21}$ ". L'attente ou la crainte de brutaux retournements des fortunes politiques fait le nid des rumeurs les plus variées car elles n'aiment rien davantage que les contextes confus et les situations instables. Pendant la période de la Restauration, certains aspirent à un retour à la stabilité dynastique de l'Ancien régime, d'autres s'attendent à de nouveaux rebondissements politiques, d'autres encore ne verraient pas d'un mauvais œil les royalistes déchirés entre deux prétendants. La Restauration, période de flou de la légitimité par excellence, est décidément une période avantageusement propice au foisonnement de rumeurs.

Certaines n'ont qu'une portée réduite : elles concernent une émotion frumentaire, une personnalité locale ou une inquiétude particulière et circonscrite. D'autres ont une ampleur nationale. Les sujets les plus inquiétants, les plus subversifs, les plus castastrophistes fournissent les rumeurs les plus vivaces. Parmi les exemples les plus signifiants, les rumeurs annonçant le retour de Napoléon de sa lointaine île d'Elbe pullulent de 1815 à $1821^{22}$. Il s'agit d'un nouveau type de rumeurs qui s'appuient sur l'espoir

19. Tulard, Jean, Les vingt Jours, Louis XVIII ou Napoléon?, Paris, Fayard, 2000, 284 p.

20. Dictionnaire des girouettes, 1815 , in- $8^{\circ}, 502 \mathrm{p}$.

21. FuRET, François, La Révolution, 1814-1880, Paris, Hachette, 1988, p. 42.

22. Sur cette question consulter MEnAGer, Bernard, Les Napoléon du peuple, Paris, Aubier, 1988, 445 p. et GuERRIN, Yann, Insubordination et opposition en Ille-et-Vilaine, Lille, éditions Septentrion, 1997, 532 p. 
d'un retour inauguré par les Cent Jours. Les bruits liés à cette aspiration ne disparaissent que très lentement. Plusieurs années après l'annonce de leur mort, Napoléon et son fils suscitent encore des espoirs chez les nostalgiques de l'Empire et des craintes chez ses adversaires. Si bien que les rumeurs impériales foisonnent. Le mystère Louis XVII se place très vite parmi les thèmes privilégiés par la population. Il s'agit, pour reprendre l'expression de Steven L. Kaplan, " de thèmes quasi-constitutifs de la mémoire et des mentalités ${ }^{23}$ ". Figurant en bonne place à côté des questions de conscription, des angoisses frumentaires, des tourments sécuritaires, des divers complots, elle apparaît comme une sous-partie de ce que l'on peut qualifier comme des rumeurs royalo-mondaines. Les rumeurs concernant les personnalités publiques existent à toutes les époques mais elles sont particulièrement nombreuses dans la première moitié du XIX ${ }^{\mathrm{e}}$ siècle car la population est avide de connaître le sort de princes tout récemment rentrés d'exil. Le jeune Louis-Charles est l'un d'eux. Héritier direct d'une illustre famille, enfant malheureux et innocente victime de la confusion révolutionnaire, le petit prince réunit autour de lui de nombreux ingrédients favorables. La force de la rumeur tient alors à l'incertitude de sa mort et à l'absence d'indice irréfutable d'identification des prétendants. Ces derniers doivent simplement élaborer un discours cohérent et présenter un âge compatible avec la date de naissance de 1785 . Le succès de cette rumeur tient bien sûr à la force du sujet. Cambacérès discerne avec clairvoyance la postérité prévisible de l'événement : " Lors même qu'il aura cessé d'exister, on le retrouvera partout, et cette chimère servira longtemps à nourrir les coupables espérances des Français traîtres à leur pays ${ }^{24}$. " La vie de Louis XVII captive l'attention au-delà même des limites raisonnables de survie d'un hypothétique survivant et le $\mathrm{xx}^{\mathrm{e}}$ siècle lui a consacré de nombreux ouvrages. Avant même sa mort, la question du sort de Louis Charles déchaîne les passions. Le destin et l'avenir de cet enfant, prisonnier au Temple, orphelin de père et bientôt de mère, tous deux guillotinés, intrigue quand il ne suscite pas haine ou pitié. Dès juillet 1793, la première rumeur le concernant déferle dans les rues de Paris : "Louis XVII aurait été enlevé par des partisans, on l'a même vu sur les boulevards, il a été porté en triomphe au château de Saint-Cloud! Il n'en faut pas moins pour qu'un attroupement se forme autour du temple ${ }^{25}$. "Inquiète, la Convention dépêche alors des agents dans la prison pour s'assurer de la présence du royal prisonnier. Pour étrangler la rumeur, les Conventionnels ordonnent de le faire descendre dans le jardin afin que les gardes puissent attester sa présence et lancer un vigoureux démenti auprès du plus grand nombre. Ainsi s'éteint la première rumeur le concernant. Mais les conditions de détention de l'enfant, les péripéties de son agonie, les mystères entourant sa mort et l'instabilité de la période révolutionnaire procurent à la ques-

23. L. KAPLAN, Steven, Le Complot de famine : histoire d'une rumeur au XVIII siècle, traduit de l'anglais par M. et J. Revel, Paris, A. Colin, 1982, 77 p.

24. Cité par Philippe Delorme, L'Affaire Louis XVII, Paris, Taillandier, p. 129.

25. Ibid., p. 92. 
tion une force inouïe ${ }^{26}$. Son décès est en effet dissimulé pendant deux jours ce qui permet toutes les suppositions postérieures d'enlèvement ou de substitution de corps. Officiellement, Louis Charles de France proclamé Louis XVII par les émigrés à la mort de son père est lui-même mort le 8 juin $1795^{27}$. Ph. Delorme ${ }^{28}$ évoque cent un faux dauphins tandis que Philippe Conrad $^{29}$ avance le chiffre d'une quarantaine. Quel que soit le chiffre avancé, il est évident que plusieurs dizaines de prétendants se sont manifestés. Ce qui témoigne de l'intérêt suscité par l'hypothèse d'une évasion et d'une substitution des corps. Les bruits gravitant autour de Louis XVII fonctionnent comme une rumeur majeure, sans cesse renaissante en différents points géographiques. C'est une rumeur polymorphe ${ }^{30}$ qui possède un tronc commun et de multiples ramifications. Elle passionne et naît presque toujours parmi le peuple. Ainsi, le prétendant de 1815 provoque d'abord l'émoi des malouines. Tout ce qui touche à des enfants possède une charge émotionnelle puissante auprès du peuple et en particulier des femmes ${ }^{31}$ : "Les femmes jouaient un rôle moteur dans les émotions provoquées par la cherté et la rareté des grains. Elles défendaient, par une sorte de réflexe biologique, la vie de leurs enfants et l'existence physique de leur foyer ${ }^{32}$. " La cruauté et l'injustice de cette mort pour raison d'État passionnent d'abord cette partie de la population. Préoccupées par le sort de leurs enfants, elles se passionnent évidemment pour celui qui a été la victime de la Terreur car " nul n'est moins sourd que celui qui veut entendre $^{33}$ ». Ce n'est que plus tard que le reste de la population prend l'affaire plus au sérieux. Ceci montre que la population réceptive ne constitue pas une masse homogène; la rumeur pénètre dans le public par le biais de certains individus : l'arrestation prend valeur de rumeur grâce à la foule des femmes qui se réunissent devant la prison. Ce sont elles qui colportent la nouvelle dans la ville puis aux alentours lors des marchés. Les poissonnières jouent un rôle mentionné par les autorités locales. Ici, ce sont surtout des femmes qui constituent ce que M.-L. Rouquette nomme " des relais primaires " ou " des guides d'opinion ${ }^{34}$ ". Elles transmettent le contenu de l'information et apportent les premières transformations et inflexions à la rumeur naissante. Dès lors, il est très difficile de suivre son cheminement avec précision. Cependant, il est indéniable que l'ensemble de la popula-

26. Les détails de son incarcération son exposée dans Philippe DELORME, L'Affaire..., op. cit.

27. CARATini, Roger, Dictionnaire des personnages de la Révolution, Paris, Le Pré aux Clercs, 1988, 557 p.

28. Philippe Delorme, L'Affaire... op. cit.

29. Philippe ConRAD, Louis XVII, l'énigme du roi perdu, Paris, 1988, éd. du May, 248 p.

30. Le qualificatif est emptunté à Edgar Morin, La Rumeur d'Orléans, Paris, Le Seuil, 1969, $235 \mathrm{p}$.

31. Comme en témoigne Jean Nicolas dans « La rumeur de Paris : rapts d'enfants en 1750 ", L'Histoire, n 40, 1981, p. 48-57.

32. Delumeau, Jean, La Peur en Occident (XIVe-XVIII siècle) Une cité assiégée. Paris, Fayard, 1978, p. 181.

33. FlEM, Lydia, "Bouche... ", art. cité, p. 15.

34. RouQueTtE, Michel-Louis, Les Rumeurs..., op. cit., p. 29. 
tion se trouve rapidement concerné par la question. La rumeur cesse d'être alimentée seulement par les femmes pour passionner toutes les couches populaires. Les autorités sont elles-mêmes saisies par le doute et proches de succomber à ses charmes. Au début de l'année 1816, le préfet d'Ille-etVilaine traduit son trouble dans plusieurs lettres adressées au ministre de la police : "Cet homme ne paraît point être fou. Je prie votre excellence de donner ordre qu'il soit conduit à Paris. Il est à remarquer qu'en parlant du roi, il dit simplement : mon oncle ${ }^{35}$. "Ces quelques lignes témoignent du désarroi et de la prudence au sein même de la préfecture! Et le 9 janvier 1816, Chateaubriand prononce un discours à la chambre des pairs qui ajoute encore au trouble et lui procure une dimension nationale :

"Qu'est-il devenu ce pupille royal laissé sous la tutelle du bourreau, cet orphelin qui pouvait dire, comme l'héritier de David; mon père et ma mère m'ont abandonné? Où est-il le compagnon des adversités, le Frère de l'Orpheline du Temple? Où pourrais-je lui adresser cette interrogation terrible et trop connue : Capet dors-tu? Lève-toi! Il se lève Messieurs, dans toute sa gloire céleste, et il vient vous demander un tombeau ${ }^{36}$ !"

Ces mots sont prononcés à l'occasion d'un débat pour la construction d'un monument commémorant la mémoire de Louis XVII. En aucun cas, ces propos ne cherchent à accréditer l'idée de la survie de Louis XVII. Mais il est difficile d'imaginer orateur et lieu plus prestigieux pour relayer une rumeur née sur le port et dans les prisons de Saint-Malo. Ce discours comporte des passages tout à fait susceptibles de déformations rumeurogènes : "Lève-toi! Il se lève Messieurs... " N'est-ce pas, à mots couverts, la confirmation de son retour? Certains pourront le penser et s'appuyer sur les paroles du prestigieux orateur. La diffusion s'effectue donc du bas vers le haut : des éléments populaires vers les élites et depuis la province bretonne vers les autres régions et Paris. La rumeur se "nourrit " alors de toutes les nouvelles pouvant la relancer, provenant de toutes les couches de la société et de toutes les régions possibles. Pendant sa diffusion, la nouvelle ne cesse d'évoluer au gré des attentes du groupe social qui le véhicule. Lorsqu'une rumeur échappe à une catégorie sociale particulière pour pénétrer l'ensemble de la société, elle peut évoluer en plusieurs versions sensiblement différentes qui s'influencent mutuellement dans un subtil jeu de rapport de force. Il en va ainsi pour l'affaire de Saint-Malo.

\section{Un Louis XVII parmi tant d'autres?}

Pour agir contre la rumeur, les autorités cherchent à confondre le prétendu Dauphin. Pour cela, il convient de lui trouver une identité. Or, les interrogatoires se heurtent à des déclarations invariables et cohérentes.

35. Le préfet au ministre de la police, le 9 janvier 1816, Arch. dép. d'Ille-et-Vilaine, 4 M 88.

36. Il est difficile de savoir si Chateaubriand est au courant des royales prétentions du prisonnier de Saint-Malo. En revanche, le contenu de son discours démontre qu'il est fidèle à la version officielle de la mort puisqu'il lui réclame un tombeau. Il n'envisage donc aucune survivance et compte bien mettre un terme à toutes les autres suppositions. 
Les tentatives locales d'identifications par l'écriture, par des souvenirs d'enfance sont toutes des échecs. Le prisonnier ne varie pas : « Je suis le dauphin de France, fils de Louis XVI, [...] on ne paraît pas me croire. Je demande à être conduit à Paris devant mon oncle qui me fera juger par les tribunaux si je ne prouve pas ce que j'avance. " Il dit avoir passé son enfance en Angleterre et déclare parler anglais. Il affirme savoir écrire mais refuse d'en faire la démonstration devant quiconque : "Oui, je sais écrire mais je ne veux le faire que devant les personnes qui peuvent me reconnaître. J'ai refusé de donner ma signature à Saint-Malo et je ne puis encore la donner ici. Je demande de nouveau à comparaître devant mon oncle ${ }^{37}$. "

Le prétendu dauphin est d'abord identifié comme étant un certain Charles Philippeau originaire de Varennes près de Saumur. Ce personnage quitte sa famille en 1807 pour répondre à la conscription. Il aurait combattu en Espagne sous les ordres du général Dupont. De retour chez les siens, il est péniblement identifié. Sa propre mère émet des doutes. Il fait ici l'expérience de la mystification en se faisant passer pour un autre. Pour les autorités, ces renseignements ne constituent qu'une piste supplémentaire qui ajoute au mystère de cet homme. Le ministre de la police intime l'ordre de transférer le prisonnier en lieu sûr. Loin de la Bretagne qui s'agite, mais pas à Paris pour éviter d'embraser la capitale où les rumeurs vont plus vite que partout ailleurs. L'extraire de la région où les rumeurs courent et se multiplient constitue une riposte habile. En même temps, le ministre prend le risque de la contagion. Pour la détention, ce sera la prison de Rouen. Là, le détenu n'est pas du tout isolé puisqu'il travaille parfois comme sabotier avec les détenus. Il faut peu de temps au " prince " pour s'entourer de nouveaux fidèles. De nombreux partisans se rallient. Il s'adjoint deux secrétaires (un faux abbé et un huissier condamné pour escroquerie) auxquels il fait rédiger le récit de son évasion de la prison du Temple, son sacre, la guerre dans les rangs royalistes et son exil pour l'Amérique. Comment se procure-t-il le roman dont il semble s'inspirer pour bâtir son récit? Nul ne le précise. Toujours est-il que de nombreux détails de sa narration proviennent manifestement d'un roman à succès paru en 1800. Dans cet ouvrage comptant quatre volumes et intitulé Le cimetière de la Madeleine, l'auteur échafaude le scénario d'une évasion du jeune roi ${ }^{38}$. Le prisonnier y puise une foule de détails qui renforcent la puissance de son récit. Une affiche clandestine est bientôt placardée dans la ville de Rouen pour réclamer sa libération. Il bénéficie en effet de complicités en ville se chargeant de diffuser ses écrits et de leur donner toute la publicité possible. Son audience se renforce. Faute de précautions élémentaires, l'étouffement de la rumeur a échoué! Après la Bretagne, elle s'empare désormais de la Normandie. Sa diffusion risque de provoquer une crise politique grave. Pour vider la rumeur de son pouvoir de nuisance, le gouvernement choi-

37. Interrogatoire mené par le préfet d'Ille-et-Vilaine sur le pseudo-Charles de Navarre le 8 janvier 1816, Arch. dép. d'Ille-et-Vilaine, 4 M 88.

38. Regnault-Warin, Le Cimetière de la Madeleine, Paris, 1800. 
sit de porter l'affaire sur la place publique. La publicité d'un procès doit faire apparaître la duplicité ou la folie du personnage.

Un rebondissement donne une nouvelle orientation à l'affaire. Brutalement, un témoin entre en scène offrant à l'accusé une nouvelle identité. Un géomètre du Maine-et-Loire affirme que l'accusé n'est pas plus le Dauphin que Philippeau mais un certain Mathurin Bruneau né en 1784.

"Orphelin de bonne heure, le jeune Mathurin avait été élevé par une sœur aînée. Dès l'âge de onze ans, il multiplie les fugues. À la faveur des troubles qui affectent alors l'ensemble de l'Ouest, il se fait passer pour le fils du baron de Vezins parti en émigration. Il est accueilli par une parente et ce n'est qu'un an plus tard que la supercherie est découverte. Transformé alors en domestique, il revient peu après chez sa sœur et son beau-frère auprès de qui il apprend le métier de sabotier. Il part à quinze ans sous prétexte d'aller faire son tour de France et il est possible qu'il ait participé, comme il l'a prétendu plus tard, à divers combats livrés par les Blancs. Il est arrêté à Paris en 1803 pour vagabondage et enfermé quelque temps à Saint-Denis [...]. Enrôlé dans l'artillerie de marine, il se retrouve en 1806 aux États-Unis puis déserte. On sait qu'il exerce successivement les professions de boulanger et de domestique par un autre déserteur venu témoigner à son procès ${ }^{39}$."

Malgré ces cinglants démentis, le personnage conserve des appuis et des partisans. Parmi eux, on peut compter la duchesse d'Angoulème, sœur du Dauphin qui dépêche des émissaires auprès du prisonnier et lui accorde un avocat. Elle est la première intéressée par la vérité et toute disposée à croire à la survie de son frère. Elle suit avec attention le procès qui débute le 9 février 1818 et qui doit permettre de faire toute la lumière grâce à l'audition de soixante-six témoins en six jours. Decazes, ministre de la police suit le déroulement de l'affaire avec attention. Il a pris soin de placer ses agents dans les rangs des spectateurs pour observer les réactions. Les rapports qui lui parviennent sont rassurants. L'attitude grossière et désinvolte de l'accusé facilite la tâche des autorités. Cependant, le contraste entre la prestation du personnage lors de son procès et sa bonne tenue habituelle font soupçonner une manœuvre des autorités pour le discréditer : certains évoquent une drogue pour lui faire perdre contrôle. D'autres décèlent plus simplement les symptômes de l'ivresse. Beaucoup s'interrogent donc sur la régularité du procès. Quoi qu'il en soit, l'énorme affluence des spectateurs suffit à démontrer l'intérêt du public pour cette affaire. Il est condamné à cinq ans de prison et transféré peu de temps après dans les geôles du mont Saint-Michel. Il y meurt très opportunément le 26 avril 1822.

39. Le contenu de ces Mémoires peut être consulté dans Philippe ConRAD, Louis XVII, L'énigme..., op. cit. Il est assez remarquable de préciser qu'en 1804 Bruneau sert sur le navire la Cybèle tout comme un autre prétendant nommé Hervagault qui figure sur le cahier d'équipage de ce même bateau en 1809. Cette coïncidence laisse perplexe mais faute de précisions, il est impossible de connaître les influences réciproques entre les deux personnages. À ce propos, consulter : Les imposteurs fameux. Ou histoires extraordinaires et singulières des hommes de néant de toutes les nations qui ont usurpé la qualité d'empereur, de roi, de prince. Terminées par celles des deux faux Louis XVII : Hervagault et Bruneau, Paris, 1818. 


\section{Les implications politiques}

À la fin de l'année 1815, en quelques semaines, les autorités comprennent qu'il convient d'accorder la meilleure attention à cette affaire. Il est notable que l'embarras domine. Comment réprimer des paroles? Il n'est pas possible de toucher l'ensemble de la population par un démenti efficace. De plus, il n'est pas possible de contrôler tous les rassemblements populaires. Or, les rencontres et les discussions dans des lieux publics sont propices à la rumeur. Les informations qui la nourrissent sont échangées au marché, au cabaret, au bal, au lavoir, partout où le nombre procure l'impunité. C'est la réponse du peuple au contrôle policier. La rumeur est un mode d'expression clandestin très difficile à maîtriser, donc dangereux pour un gouvernement soucieux de contrôler les opinions. Dans une société bridée par la censure et le contrôle des mouches de la police, la rumeur tient lieu de défoulement. Elle se situe à la convergence de la curiosité et de l'opposition politique. Elle répond aussi à une attente, à un espoir.

Ce n'est que plus tard, avec le procès, que toute la société se passionne pour l'hypothèse malouine et que le ministère de la police y accorde toute l'attention nécessaire. Le gouvernement accepte alors de donner une large publicité à l'affaire. Il prend le risque de la diffusion. Dès lors, elle gagne en ampleur. Les autorités prennent à témoin l'opinion de tout le pays et entendent démontrer l'imposture. Or, plus une rumeur est répandue plus elle se crédibilise : elle vit par elle-même en éliminant tout ce qui la contredit. Elle est plus forte que la vérité! Avec la publicité d'un procès, il s'agit désormais d'un sujet de conversation où chacun peut apporter son commentaire. Les autorités comptent sur la raison et le bon sens pour faire triompher leur vérité. Plus que jamais, les individus deviennent à la fois récepteurs puis émetteurs relayant sans fin les bruits les plus variables. Ceux qui participent à une rumeur de ce genre sont à la fois des oreilles qui écoutent et des bouches qui répètent : double motif d'arrestation! La population se lance souvent sans retenue dans ces bruits et nouvelles car ils lui permettent de se défouler. Les colporter c'est tout à la fois renforcer son intégration au sein d'un groupe social et en même temps partager son anxiété ou son opposition.

R. Knapp évoque les "rumeurs de désir ", celles qui expriment les espoirs de la population et se traduisent par la diffusion d'informations satisfaisantes. La rumeur de Saint-Malo se rattache à cette catégorie puisqu'il s'agit de croire en la " résurrection » d'un enfant. Mais elle s'apparente aussi à une autre catégorie définie par Knapp : la " rumeur d'agression ", celle qui cherche à nuire à une partie de la population ou à une élite dirigeante $^{40}$. Il s'agit donc d'une rumeur à double détente. Comment l'espoir de la survie d'un héritier royal peut-il constituer un crime? La police dis-

40. La seconde famille correspond aux rumeurs de crainte et d'anxiété qui se greffent sur une angoisse populaire. La rumeur que nous étudions ici ne se rattache pas directement à cette catégorie. Robert KNAPP, art. cité. 
cerne assez confusément les dangers de ce genre de situation : la revendication du titre de Dauphin signifie la négation de la légitimité du pouvoir de Louis XVIII. La rumeur est donc répréhensible dans ses non-dits. Mais les moyens répressifs dont les autorités disposent sont très minces.

En même temps, les autorités de cette époque, toujours très inquiètes des mouvements populaires, ne demandent qu'à accorder grand crédit à ce genre d'affaire. En effet, selon Jean Delumeau, il est « impossible, s'agissant du moins de la civilisation pré-industrielle, de séparer rumeur et sédition $^{41}$ ". La police cherche à connaître le contenu et les relais principaux car elle craint constamment des dérives politiques : briser une rumeur c'est peut-être éviter une complication vers de dangereuses séditions. Derrière la rumeur, l'administration policière craint toujours de trouver un mystérieux complot impliquant de très nombreuses complicités. Sitôt connus, les suspects sont arrêtés. La fébrilité des autorités à la moindre rumeur transpire au travers de brutales recrudescences des patrouilles, des interrogatoires, des policiers qui rôdent dans les lieux de rassemblement. Toute cette activité policière confère une force nouvelle aux bruits qui circulent. Le " démon du soupçon ${ }^{42}$ ", la crainte permanente du complot font le jeu de la rumeur. Pour la contrôler, les autorités cherchent à agir en plusieurs directions.

En réalité, les conditions pour lutter sont alors loin d'être réunies. Il faudrait restaurer la confiance et asseoir l'autorité des dirigeants. Nous n'insisterons pas sur la fragilité de la situation politique des années 1815-1816! En outre, il est depuis longtemps impossible d'exposer la dépouille mortelle du vrai Dauphin. Faute de moyens de communication touchant toute la population, il n'est pas plus envisageable de diffuser des démentis efficaces. Le procès est donc le seul recours qui puisse satisfaire l'avidité du public en discréditant l'accusé et en diffusant des informations autorisées. Mais démentir la rumeur c'est déjà lui donner une forme de reconnaissance officielle. Il conviendrait davantage de détourner l'attention de la population en lançant d'autres rumeurs plus intéressantes que la précédente. Ce n'est pas sans risque car une fois lancées elles échappent à leurs initiateurs. La solution d'un procès public qui est choisi dans le cas présent n'est pas si mauvaise : elle offre une confrontation au grand jour permettant de combattre les principaux arguments. Si un procès est ici possible c'est que la rumeur peut être personnifiée ce qui n'est pas le cas le plus fréquent.

La rumeur naît donc sur un fond d'inquiétude et de dissolution de l'autorité ou de sa légitimité. En cela la rumeur de 1815 est tout à fait le reflet de l'ambiance politique des débuts de la Restauration. Elle n'est pas que cela. Elle correspond à un mouvement beaucoup plus profond. Une véritable lame de fond qui est très révélatrice des mentalités populaires de cette époque : la question de l'enfant royal passionne plusieurs générations

41. Delumeau, Jean, La Peur..., op. cit.

42. D'après le titre d'un article de Maurice GAUCHET, " Le démon du soupçon ", L'Histoire, $\mathrm{n}^{\circ} 88,1985$, p. $48-57$. 
de la première moitié du XIX ${ }^{\mathrm{e}}$ siècle. La rumeur en question doit donc être replacée dans un mouvement de plus grande ampleur. Puisqu'elle réapparaît plusieurs fois sous les traits d'un nouveau prétendant, elle est le fruit d'une longue préparation mentale. Elle se rattache à des souvenirs et des aspirations antérieures : elle ne reprend jamais à partir de rien. Plusieurs prétendants au titre de fils de Louis XVI provoquent l'émotion du public. "La rumeur ne se réduit pas non plus aux éruptions soudaines passagères mais récurrentes d'un désordre des corps et des esprits. Elle semble au contraire produire des effets cumulatifs, même si les contemporains les ont probablement ignorés ${ }^{43}$. " Les grandes rumeurs sont identifiables par leur capacité à captiver un large public et à renaître en s'adaptant aux changements de l'époque et du lieu. Il faut préciser qu'à la fin de l'année 1816, le temps de M. Bruneau est passé, la rumeur du Dauphin survivant s'étiole en Bretagne et en Normandie pour surgir ailleurs. Dès 1816, un laboureur de Gallardon près de Chartres prétend avoir des informations capitales sur Louis XVII et obtient une entrevue avec Louis XVIII. L'affaire reste sans suite mais montre combien la question du sort de cet enfant procède du fantasme collectif ${ }^{44}$. Quelques années plus tard. La rumeur reprend au nord de l'Italie quand un certain Claude Perrin est arrêté à Modène (1820). La rumeur présente alors les mêmes caractéristiques. Elle s'est déplacée de plusieurs centaines de kilomètres.

Il ne nous appartient pas ici de poursuivre sur tous les prétendants au titre de survivant de la prison du Temple. Notons cependant la vivacité d'une rumeur capable de s'incarner dans plusieurs dizaines de personnages. Chacun lui prête vie. Le célèbre Naundorff est le dernier grand prétendant. Cependant, Yves-Marie Bercé dans Le roi caché, considère qu'il ne possède pas la même envergure que $\mathrm{M}$. Bruneau qui reste le seul à présenter une véritable surface politique ${ }^{45}$. Il formule en effet ses prétentions à un moment d'inquiétude populaire. Il rencontre une audience telle qu'il constitue un danger pour le pouvoir. À partir de 1820, le contexte politique s'est modifié. L'opinion se passionne alors pour une autre affaire extraordinaire : la naissance du duc de Bordeaux surnommé immédiatement « l'enfant du miracle ", le fils posthume du duc de Berry. Après 1830, avec la chute des Bourbons, les querelles survivantistes s'atténuent. Naundorff est probablement le dernier avatar d'une rumeur réelle mais sans grande implication politique car elle ne peut pas se prolonger indéfiniment : un personnage né en 1785 ne peut survivre éternellement! La rumeur s'étiole faute de vraisemblance aux environs du Second Empire. Elle correspond finalement à la première moitié du XIX ${ }^{\mathrm{e}}$ siècle. Mais la question garde valeur d'énigme historique pendant tout le $\mathrm{xx}^{\mathrm{e}}$ siècle. Ce n'est qu'à l'extrême fin de ce siècle que la certitude permet une fois pour toutes de balayer les

43. KaPlan, Steven L., Le Complot..., op. cit.

44. Pendant l'Empire, une insurrection royaliste est menée par un nommé Fruchart dit Louis XVII. Cité par Pierre FAuciLle, Une chouannerie flamande, 1813-1814, 1905.

45. Berce, Yves-Marie, Le Roi caché, Paris, Fayard, 1990, 483 p. 
doutes qui restaient : le 19 avril 2000, les séquences ADN analysées à partir du cœur prélevé sur le cadavre de l'enfant mort au temple sont les mêmes que celles des cheveux de Marie-Antoinette ${ }^{46}$. Le petit garçon mort en juin 1795 était donc bien son second fils, Louis-Charles, duc de Normandie devenu Louis XVII à la mort de son père. Les prétendants étaient donc tous des imposteurs. Les rumeurs reposaient toutes sur de fausses prétentions. Il reste qu'elles ont bien existé et profondément agité le royaume tout particulièrement durant les périodes de fébrilité politique.

La portée politique ne peut en effet nous échapper. Pas plus qu'au souspréfet de Fougères qui écrit :

" N'est-ce pas une machination nouvelle? Buonaparte est parti, Napoléon Il n'a pas pris, le duc d'Orléans n'a pas voulu, ne voudrait-on pas essayer si un fantôme ne pourrait pas exciter de nouveaux troubles. Ce qu'il y a de certain, c'est que les gens les moins tendres pour l'auguste famille qui nous gouverne se sont fort attendris sur le sort de cet infortuné jeune homme s'il est réellement celui qu'on croit mort ${ }^{47}$."

On ne peut manquer de remarquer que la rumeur en question est révélatrice d'une opposition au nouveau pouvoir en place après l'Empire. La rumeur révèle une partie cachée des opinions d'une frange populaire de la population française sous la Restauration. Cette partie de l'opinion trouve alors un moyen d'exprimer ses doutes. En cela, la rumeur constitue un poste d'observation de mentalités populaires toujours surveillées mais trop rarement consultées.

46. Consulter le site internet, http://www.chez.com/louis17/odyssee-coeur.htm ou Philippe Delorme, Louis XVII, la vérité. Sa mort au temple confirmée par la science, Paris, Pygmalion, 2001, 278 p. ou Louis HASTIER, La double mort de Louis XVII, Paris, Flammarion, 2001, 304 p.

47. Sous-préfet à Fougères au préfet à Rennes, le 24 décembre 1815, Arch. dép. d'Illeet-Vilaine, 4 M 88. 
RESUME

À Saint-Malo, en décembre 1815, un inconnu arrêté par la police se prétend le fils de Louis XVI. C'est le point de départ d'une rumeur qui submerge la Bretagne puis la Normandie et enfin toute la France.

Au-delà des prétentions d'un pseudo Louis XVII, c'est d'une rumeur à portée politique dont nous traitons dans cet article.

\section{ABSTRACT}

Saint-Malo, december 1815: a stranger who claim to be the son of Louis XVI was arrested by the police. This is the starting point of a rumour which spread over Brittany, Normandy ultimately the whole of France.

Beyond the claims of a self proclaimed Louis XVII, this article will deal with a rumour that is politically-based. 